\title{
Antithymocyte globulin and cyclosporin: standard of care also for older patients with aplastic anemia
}

\author{
Andrea Bacigalupo \\ Fondazione Policlinico Universitario A. Gemelli IRCCS, Universita' Cattolica del Sacro Cuore, Rome, Italy \\ E-mail:apbacigalupo@yahoo.com
}

doi:10.3324/haematol.2018.207167

T reatment of aplastic anemia (AA) is challenging because cytopenia exposes patients to infectious and hemorrhagic complications, and immunosuppressive agents may further increase the risk in the short term: this is particularly true in older patients, in whom age, per se, is a risk factor. For this reason, I believe the study published in this issue on behalf of the French Cooperative Study Group for Aplastic Anemia, ${ }^{1}$ on 88 patients over the age of 60 years, given immunosuppressive therapy (IST), is clinically relevant and useful on a practical level. The authors identified 184 treatments, including the standard combination of anti-thymocyte globulin and cyclosporine-A (ATG-CsA). Responses were significantly better in those that had followed an ATG-CsA regimen; overall response rate was $70 \%$ after first-line treatment and the overall 3 -year survival was an excellent $74 \%$. The Authors conclude that age, per se, is not a limiting factor for standard immunosuppressive treatment of patients with AA.

Treatment of severe aplastic anemia (SAA) patients over the age of 60 is problematic, and transplant studies claiming good results in older patients ${ }^{2}$ contain only very few patients over the age of 60: in the study by Shin and coworkers, which suggests that transplant outcome is comparable in patients younger or older than 40 years, the maximum patient age was 63 years. ${ }^{2}$ The cyclophosphamide (CY) dose-finding study by Anderlini and coworkers on patients receiving an alternative donor transplant, which showed over $90 \%$ one-year survival for the low dose CY group, had only a handful of patients over the age of 40, and the median age overall was 21 years. ${ }^{3}$ More recently, a European Group for Blood and Marrow Transplantation (EBMT) study showed that the outcome of transplant for SAA patients over the age of 40 remains unsatisfactory ${ }^{4}$ and has not improved in the past decade. Also, in patients with leukemia, age is a predictive factor, but transplant mortality is much lower than in SAA patients; a recent study in patients over the age of 70 years reported a transplant mortality of $20 \%{ }^{5}$ Therefore, age has a stronger impact on survival after transplantation in SAA than leukemia, for reasons which are not clear. Currently, transplantation over the age of 60 is rarely taken into consideration by transplant centers: in the EBMT study 2010-2015, 95 grafts there performed on patients over the age of 60 (16 transplants/year in Europe). ${ }^{4}$

Immunosuppressive treatment is also strongly age-dependent: in a prospective randomized EBMT study, the 6-year survival was $100 \%$ for patients $<20$ years old, $92 \%$ for patients aged $20-40$ years, $71 \%$ for those aged $40-60$, and $56 \%$ for patients older than 60 years. ${ }^{6}$ Two recent retrospective analyses have addressed the issue of first-line treatment in a large number of SAA patients with either horse ATG-CsA (in 465 patients) ${ }^{7}$ or rabbit ATG-CsA (in 955 patients). ${ }^{8}$ The one year cumulative incidence of response was $65 \%$ for both horse or rabbit ATG, but patients over 60 years of age had lower response rates. ${ }^{7.8}$ As for survival, the horse ATG-CsA study shows a 5 -year survival of $70 \%$ for patients over the age of 40 , compared to $90 \%$ for young patients. ${ }^{7}$ The rabbit ATG-CsA study shows a similar 5-year survival for patients aged 40-60 $(80 \%)$, but over the age of 60 , survival at 5 years is in the order of $50 \%$, compared to $90 \%$ for younger patients. ${ }^{8}$ Therefore, also after ATG-CsA, age remains one of the major predictors of response and survival.

The French study by Contejean and coworkers ${ }^{1}$ compares
Response to IST in older patients with AA

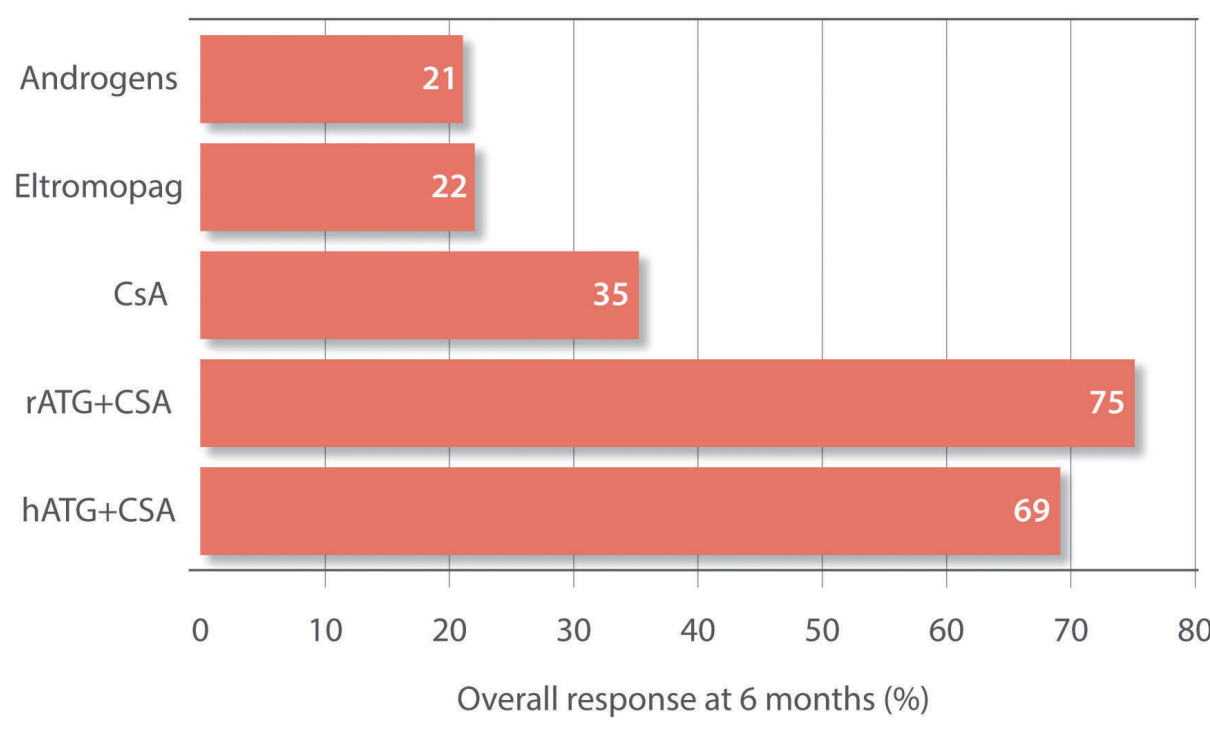

Figure 1. Proportion of patients responding to immunosuppressive therapy (IST). hATG: horse anti-thymocyte globulin; rATG: rabbit ATG; CsA : cyclosporine A. 
favourably with these two large international reports: response rate to ATG+CsA at 6 months was $70 \%$ compared to $35 \%$ for patients receiving CsA alone, $22 \%$ for eltrombopag alone, and $21 \%$ for androgens alone (Figure 1). Overall response rates in patients receiving these different treatment options is outlined in Figure 1, which highlights a superior response rate with the ATG+CsA combination. Figure 1 also shows there was no difference in response for patients in this older patient population receiving horse (hATG) versus rabbit ATG (rATG), which is in keeping with two large recently published real-life studies., ${ }^{7,8}$ Also, survival was comparable in patients receiving horse or rabbit ATG. The higher response rate of patients receiving ATG+CsA did not translate to a significantly improved survival compared to other regimens, as already shown in an EBMT study; ${ }^{9}$ possibly due to second treatment or improved supportive care. Further support to long-term survival also for non-responders comes from the rabbit ATG study; it showed that the 10-year survival of AA patients, classified at 6 months as non-responders to a first course of rATG+CsA, was comparable whether patients were then allografted (64\% survival) or not $\left(60 \%\right.$ survival). ${ }^{8}$ Late responses, improved supportive care, and second treatments could possibly explain the outcome of non-allografted patients.

In conclusion, $\mathrm{ATG}+\mathrm{Cs}$ A remains the treatment of choice for patients with $\mathrm{AA}$, also for those over the age of 60 ; it should be preferred over the administration of CsA with or without androgens or eltrombopag, with or without CsA. Whether the addition of eltrombopag to ATG-CsA first line will further improve the outcome, as recently suggested, ${ }^{10}$ will be determined by an ongoing prospective randomized trial (RACE trial, EBMT). This study also confirms that in real-life analysis, horse or rabbit ATG produce almost identical response rates and survival, also in older AA patients.

\section{References}

1. Contejean A, Resche-Rigon M, Tamburini J et al. Aplastic anemia in the elderly: a nationwide survey on behalf of the French Reference Center for Aplastic Anemia. Haematologica, 2018;104 (2):256-262.

2. Shin SH, Jeon YW, Yoon JH, et al. Comparable outcomes between younger ( $\leqslant 40$ years) and older ( $>40$ years) adult patients with severe aplastic anemia after HLA-matched sibling stem cell transplantation using fludarabine-based conditioning. Bone Marrow Transplant. 2016;51(11):1456-1463

3. Anderlini P, Wu J, Gersten I, et al. Cyclophosphamide conditioning in patients with severe aplastic anaemia given unrelated marrow transplantation: a phase 1-2 dose de-escalation study. Lancet Haematol. 2015;2(9):e367-75.

4. Giammarco S, Peffault de Latour R, Sica S, et al. European Group for Blood and Marrow Transplantation Severe Aplastic Anemia Working Party. Transplant outcome for patients with acquired aplastic anemia over the age of 40: has the outcome improved? Blood. 2018;131(17):1989-1992

5. Al Marki MM, Nathwani N, Yang D, et al. Melphalan based reduced intensity conditioning is associated with favorable disease control and acceptable toxicities in patients older than 70 years with hematologic malignancies, undergoing allogeneic stem cell transplantation. Biol Blood and Marrow Transpl. 2018;24(9):1828-1835

6. Tichelli G, Schrezenmeier H, Socie G, et al. A randomized controlled study in patients with newly diagnosed severe a aplastic anemia receiving antithymocyte globulin (ATG), cyclosporine, with or without GCSF: study of the SAA Working Party of the European Group for Blood and Marrow Transplantation. Blood. 2011;117(17):4434-4441

7. Peffault de la Tour R, Tabrizi R, Marcais A, et al. Nationwide survey on the use of horse antithymocyte globulins (ATGAM) in patients with acquired aplastic anemia: A report on behalf of the French Reference Center for Aplastic Anemia. Am J Hematol. 2018;93(5):1-8

8. Bacigalupo A, Oneto R, Schrezenmeier H, et al. First line treatment of aplastic anemia with thymoglobuline in Europe and Asia: Outcome of 955 patients treated 2001-2012. Am J Hematol. 2018;93(5):643-648

9. Marsh J, Schrezenmeier H, Marin P, et al. Prospective randomized multicenter study comparing cyclosporin alone versus the combination of antithymocyte globulin and cyclosporin for treatment of patients with nonsevere aplastic anemia: a report from the European Blood and Marrow Transplant (EBMT) Severe Aplastic Anaemia Working Party. Blood. 1999;93(7):2191-2195

10. Townsley D, Scheinberg P, Winkle T, et al. Eltrombopag added to standard immunosuppression for aplastic anemia. New Engl J Med. 2017; 376(16):1540-1545.

\title{
Milk and the "Grandmother Effect"- a new contribution to the legacy of Ray Owen
}

\author{
William J. Burlingham \\ University of Wisconsin, Madison, WI, USA \\ E-mail:burlingham@surgery.wisc.edu
}

doi:10.3324/haematol.2018.207340

T he article by Schonewille et al., in the September 2018 edition of Haematologica, ${ }^{1}$ tests the theory originally proposed by Owen et al. [PNAS, 19542] that a "Grandmother Effect" can protect the offspring from hemolytic disease of the fetus and newborn (HDFN) [see illustration, Figure 1]. This disease is caused by maternal antibodies formed against the baby's red blood cells (rbc). One should note here that, thanks to the research of Owen and others in the 1950s, anti-Rhesus $\mathrm{D}(\mathrm{RhD})$ antibody (aka "Rhogam") prophylaxis began to be practiced for all pregnant women who are $\mathrm{RhD}$ - bearing an $\mathrm{RhD}+$ fetus. The absence of such therapy prior to 1954 enabled Owen and colleagues to determine that, when the grandmother had $\mathrm{RhD}$, the mother who was $\mathrm{RhD}$ - and had thereby already been "naturally" exposed to this antigen in utero and as a newborn via breast-feeding, was rendered incapable (for the most part) of producing antibody to an $\mathrm{RhD}$ encountered in her adult life as the mother of a child sired by her $\mathrm{RhD}+$ husband. Subsequent to the routine application of Rhogam (anti-RhD prophylaxis) in the 1960's, the incidence of hemolytic disease of the newborn was greatly decreased, although not completely eliminated. Briefly, Rh- mothers are given a bolus of Rhogam at 28 weeks of pregnancy. If the baby that is born is $\mathrm{Rh}+(\mathrm{a}$ 50:50 chance, if her husband is $\mathrm{RhD}$ heterozyogous), she will receive a second dose, 72 hours after giving birth. This procedure has revolutionized obstetrics and made it possible for healthy $\mathrm{Rh}+$ babies to be born from multiple pregnant $\mathrm{Rh}$ - women.

While a boon to Ob/Gyn medicine, this highly effective clinical practice made it nearly impossible for others to 